\title{
Changes and Clinical Significance of Detailed Peripheral Lymphocyte Subsets in Evaluating the Immunity for Cancer Patients
}

This article was published in the following Dove Press journal: Cancer Management and Research

\author{
Jinrong Qiu',* \\ Fuping Zhou' ${ }^{1} *$ \\ Xinchun $\mathrm{Li}^{2}, *$ \\ Sufang Zhang ${ }^{2}$ \\ Zhuo Chen ${ }^{2}$ \\ Zenghui $\mathrm{Xu}^{2}$ \\ Gaoxiong $\mathrm{Lu}^{2}$ \\ Zhi Zhu ${ }^{3}$ \\ Na Ding ${ }^{2}$ \\ Jinxing Lou ${ }^{4}$ \\ Zhenlong $\mathrm{Ye}^{2}$ \\ Qijun Qian ${ }^{1,2,4}$ \\ 'Department of Biotherapy, Eastern \\ Hepatobiliary Surgery Hospital, \\ The Second Military Medical University of \\ Chinese PLA, Shanghai, People's Republic \\ of China; ${ }^{2}$ Shanghai Baize Medical \\ Laboratory, Shanghai, People's Republic \\ of China; ${ }^{3}$ Department of Pathology, \\ Changhai Hospital, The Second Military \\ Medical University of Chinese PLA, \\ Shanghai, People's Republic of China; \\ ${ }^{4}$ Shanghai Mengchao Cancer Hospital, \\ Shanghai, People's Republic of China
}

*These authors contributed equally to this work
Correspondence: Qijun Qian; Zhenlong Ye Shanghai Baize Medical Laboratory, Yuanguo Road, Shanghai 201805, People's Republic of China

Email qian@shcell.org; yezl@shcell.org
Objective: The evaluation of lymphocyte subsets is widely regarded as an important factor for monitoring tumor progression and response to therapy. This study was designed to establish a comprehensive and detailed assessment of peripheral lymphocyte subsets with a multi-parametric flow cytometry assay for response prediction and prognosis evaluation of cancer patients.

Methods: Peripheral blood samples collected from 40 cancer patients and 23 age- and sexmatched healthy volunteers were tested for 29 lymphocyte subsets by flow cytometry. The univariate analysis was applied to establish the reference interval of healthy samples, and the ratio and proportion of 29 lymphocyte subsets between patient samples and healthy controls were compared to evaluate their clinical significance by Mann-Whitney $U$-test model.

Results: The reference ranges of 29 lymphocyte subsets were established with a normal distribution and no significant differences were observed between genders. Compared with healthy control group, lower proportion and ratio of specific parameters, such as Naïve Th cells $(p<0.01)$, Naïve Tc cells $(p<0.01)$, CM (central memory) Tc cells $(p<0.01)$, Naïve T cells/ Memory T cells $(p<0.001)$, Naïve T cells/EM (effector memory) T cells $(p<0.001)$ and Naive Th cells/Memory Th cells $(p<0.001)$, and higher proportion and ratio of EM Th cells $(p<0.001)$, EM Tc cells $(p<0.01)$, effector Tc cells $(p<0.05)$, EM Th cells/CM Th cells $(p<0.01)$ and EM Tc cells/CM Tc cells $(p<0.01)$, as well as Breg $(p<0.001)$, B cells $(p<0.05)$ and CD16-NK cells $(p<0.001)$ were found in cancer cohorts.

Conclusion: This study suggests that the changes in certain lymphocyte subsets might be helpful to evaluate the immunity of cancer patients, and holds great potential for clinical application.

Keywords: solid tumor, lymphocyte subsets, clinic significance, reference intervals, flow cytometry

\section{Introduction}

Malignant cancers are the primary causes of death in the world nowadays. The standard treatments include surgery, radiotherapy and chemotherapy. Immune checkpoint inhibitors and CAR-T (chimeric antigen receptor-modified T) therapy have been reported to be the breakthrough therapeutics that can extend the overall survival time of patients with malignant tumors who could not be cured by the conventional therapies. ${ }^{1,2}$ Carcinoma is generally considered as the consequence of an imbalanced immune system. Cancer cells escape from the immune surveillance, proliferate promptly and express unique biomarkers that trigger innate and adaptive 
immune responses. ${ }^{3}$ The subsets of T cells, B cells and NK cells have been revealed to play a critical role in assisting in (Treg, Breg) or restraining $(\mathrm{CD} 4+\mathrm{T}, \mathrm{CD} 8+\mathrm{T})$ the immune escape. ${ }^{4-6}$ And they were widely regarded as the predictive or prognostic indicators for patients with malignant solid tumors. ${ }^{7-10}$

Clinically, immunophenotyping of peripheral blood plays an important role in the auxiliary diagnosis of lymphomas. ${ }^{11}$ The prognostic values of peripheral Naïve CD4+T/Memory CD4+T in non-small cell lung cancer have been investigated by Peng et al. ${ }^{12}$ Lan et $\mathrm{al}^{13}$ found the correlation between imbalanced Treg/Th17 and HCC (hepatocellular carcinoma) progression and prognosis. Therefore, it is imperative to know the immune system status for patients with malignant solid tumors. However, the HIPC (Human Immune Phenotyping Consortium) panel of T cells, Treg, Th1/2/17, B cells, and NK/dendritic cells/monocytes has limitations to evaluate the immune function of cancer patients for clinical monitoring and prognosis. $^{14}$

In this article, we intend to figure out whether the above indicators are applicable to a variety of patients with malignant solid tumors, and to explore more accurate, reliable and novel indicators. This study is aimed to make a comprehensive and detailed assessment of human lymphocyte subsets in peripheral blood by a multi-parametric flow cytometry assay and to investigate the useful indicators in early diagnostics and prognosis for patients with malignant tumors. In addition, the reference intervals in adults aging from 27 to 62 were also provided.

\section{Methods}

\section{Subjects/Patient Selection}

Twenty-three age- and sex-matched healthy volunteers were selected. Those with tested HIV, systemic infection, connective tissue diseases, abnormal tumor markers or cancers were excluded. ${ }^{35}$ Their average age was 41.89 ranging from 27 to 62 . Informed consent was obtained from all subjects. Forty patients with 15 types of solid tumors were free from therapies that may influence patients' immune status, including esophageal carcinoma (2 males), colorectal carcinoma (1 male, 1 female), pancreatic carcinoma (1 male, 3 females), ovarian carcinoma (1 female), liver carcinoma (13 males, 1 female), stomach carcinoma (1 male), renal carcinoma ( 2 males, 1 female), lung carcinoma ( 3 males, 1 female), breast carcinoma (2 females), chondrosarcoma ( 1 female), laryngeal carcinoma (1 male), bile duct carcinoma ( 1 male, 1 female), lymphoma (1 male), nasopharynx carcinoma (1 male), LSCC (Laryngeal squamous cell carcinoma) (1 male). The present study was approved by the ethics committees of Eastern Hepatobiliary Surgery Hospital in Shanghai, China and performed in accordance with relevant guidelines and regulations.

\section{Assays for Lymphocyte Immunophenotyping}

Fresh peripheral blood samples obtained from healthy donors and patients were collected in EDTA anticoagulation tubes before testing. For the analysis of lymphocyte immunophenotyping, 3 panels with the monoclonal antibodies cocktail were designed to identify 29 lymphocyte subsets (Table 1). Initially, $100 \mu \mathrm{L}$ blood was mixed with the specific antibody cocktail in each panel and incubated 25-30 min in the dark at room temperature. By using OptiLyse C Lysing Solution (Beckman Coulter, USA), red blood cells in the mixture were lysed and then washed twice with phosphate-buffered saline (PBS). The residual nucleated cells were resuspended with $300 \mu \mathrm{L}$ PBS and analyzed by flow cytometry (Navios, Beckman Coulter, USA) and the percentages of lymphocyte subsets were calculated by Navios Software 1.3. To evaluate the panel, the fluorescence minus one (FMO) test was performed.

\section{Statistical Analyses}

Statistical analyses were performed using IBM Statistics software, version 20.0 (IBM Corporation, Armonk, NY, USA). $P<0.05$ was defined as statistically significant. Reference intervals were calculated based on the recommendations of the International Federation of Clinical Chemistry (IFCC). ${ }^{36}$ The Kolmogorov Simonov test was

Table I Antibody Composition of Three Panels for Differentiating Lymphocyte Subsets

\begin{tabular}{|c|c|c|c|c|c|c|c|c|}
\hline Fluorochrome & FITC & PE & PerCP-Cy ${ }^{\mathrm{TM}} 5.5$ & APC & PE-Cy ${ }^{\mathrm{TM}} 7$ & APC-Cy ${ }^{\mathrm{TM}} 7$ & BV42I & BV5 IO \\
\hline Panel I & TCR $\gamma \delta$ & TCR $\alpha \beta$ & CD4 & CD45RA & & CD8 & CDI97 & CD3 \\
\hline Panel 2 & CDI27 & CDI96 & CD4 & CDI83 & CD56 & CDI6 & CD25 & $\mathrm{CD} 3$ \\
\hline Panel 3 & CDI9 & & CD24 & CD5 & & & CD38 & CD27 \\
\hline
\end{tabular}


performed to determine data distribution. Relationships between lymphocyte subsets within genders were determined by Student's $t$-test. Differences of lymphocyte subsets between cancer patients and controls were compared by using Mann-Whitney $U$-test model.

\section{Results}

\section{Establishment of Reference Values for Lymphocyte Subsets}

A total of 23 healthy Chinese volunteers including 9 males and 14 females were recruited to evaluate the function of the immune system and to establish reference intervals for human lymphocyte subsets by multi-parametric flow cytometry. Three panels for 29 lymphocyte subsets were differentiated by flow cytometry (Figures 1-3). Panel 1 included 1 ratio and 14 lymphocyte subsets, in which
$\mathrm{T}$ cell subsets were divided into three logical hierarchies. The first subsets were the total $\mathrm{T}$ cells identified by CD3 from lymphocytes, and then they were differentiated into TCR $\alpha \beta$ and TCR $\gamma \delta$ cells based on the types and specific functions of the surface receptor. $\mathrm{T}$ cells can also be divided into subsets of Th (CD4+CD8-), Tc (CD4-CD8+) and DNT (CD4-CD8-). In immune response, the functional $\mathrm{T}$ cell subsets, e.g. Th and Tc, were further divided into Naïve Th/Tc, Effector Th/Tc, Center memory $\mathrm{Th} / \mathrm{Tc}$ and Effector memory $\mathrm{Th} / \mathrm{Tc}$ that were identified by CD45RA and CD197. Panel 2 had 8 lymphocyte subsets, including $4 \mathrm{~T}$ lymphocyte subsets, NK cells together with its 2 subsets, NKT cells, CD3-CD56-CD16+ cells. There was two paralleled logical hierarchy, one was $\mathrm{T}$ lymphocyte subsets logical line which identified the Treg (CD25+CD127dim/-), Th1 (CD196-CD183+), Th2
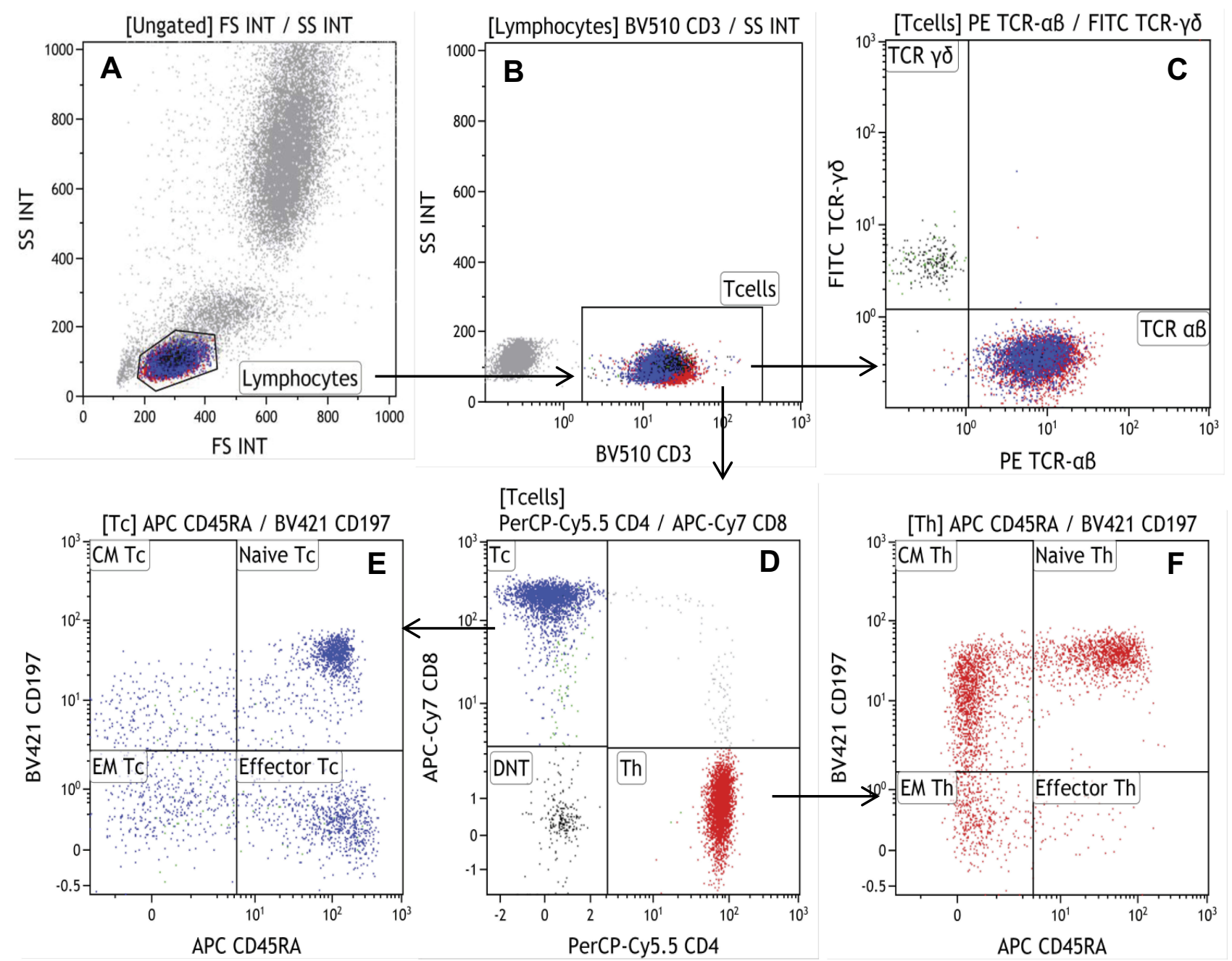

Figure I Gating strategy of T cell subsets (Panel I). Gating the lymphocytes by physical characteristics (A). T cells were identified by CD3 staining (B), and TCR $\boldsymbol{\alpha} \boldsymbol{\beta}, \mathrm{TCR} \boldsymbol{\gamma} \boldsymbol{\delta}$ were gated from T cells (C). CD4 and CD8 stainings were used to gate Th, Tc (D), Effector memory subsets of Th and Tc can be further divided into CD45RA and CDI 97 (E, F). 

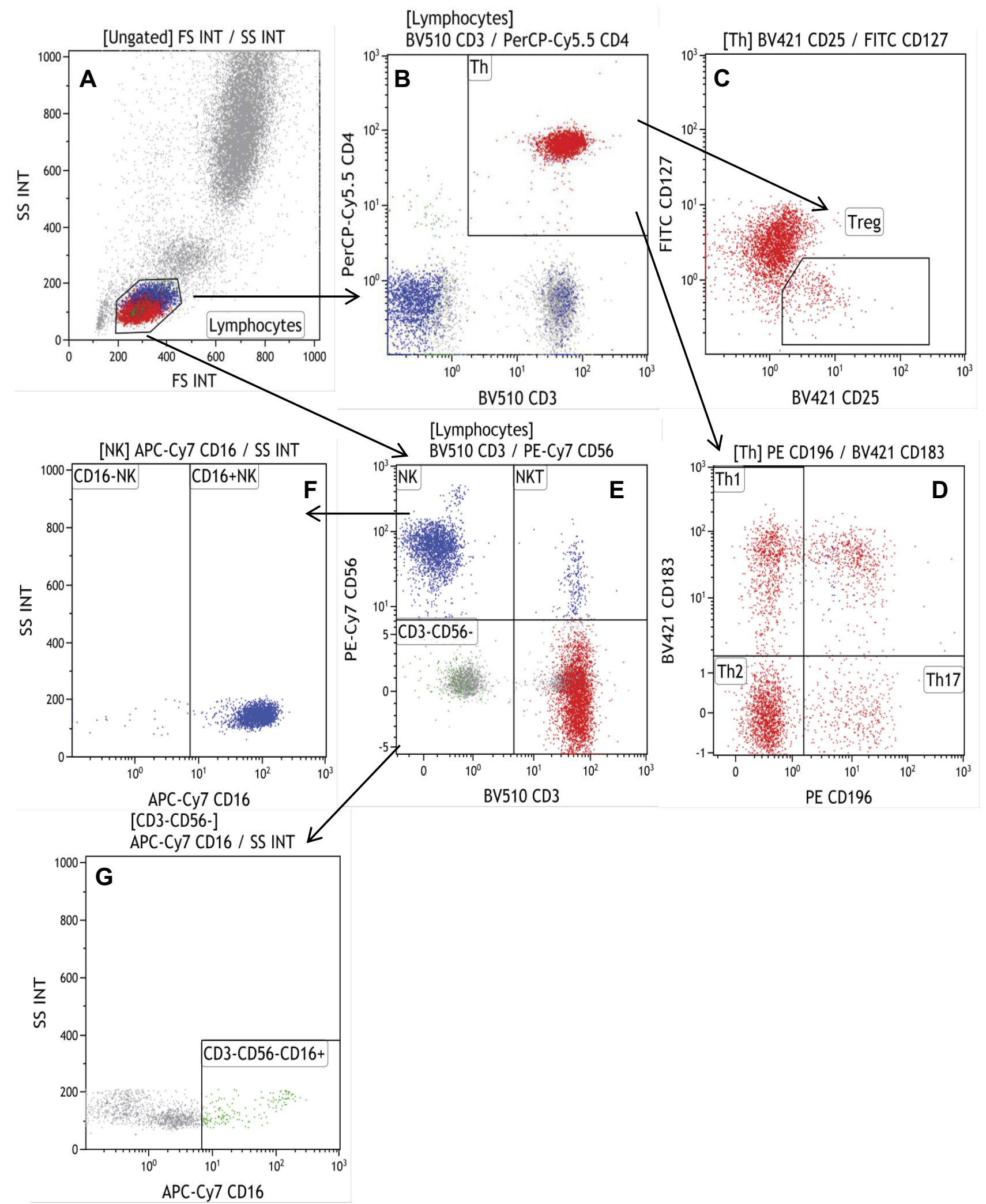

Figure 2 Gating strategy of NKT and T, NK cell subsets (Panel 2). Lymphocytes were gated according to their size and granularity in forward (FS INT)/side scatter (SS INT) (A). Treg can be identified with CD25 expression and low or negative expression of CDI27 (C). ThI, Th2 and Th17 were gated from Th (B) that can be identified by CDI96 and CDI83 expression (D), the difference between NK and NKT cells was identified whether there was CD3 expression (E), the NK subpopulation can be divided with CDI6 staining (F), CD3-CD56-CD I6+ cell population (G) may be associated with $\mathrm{HCV}$ infection or AIDS (autoimmune diseases). 

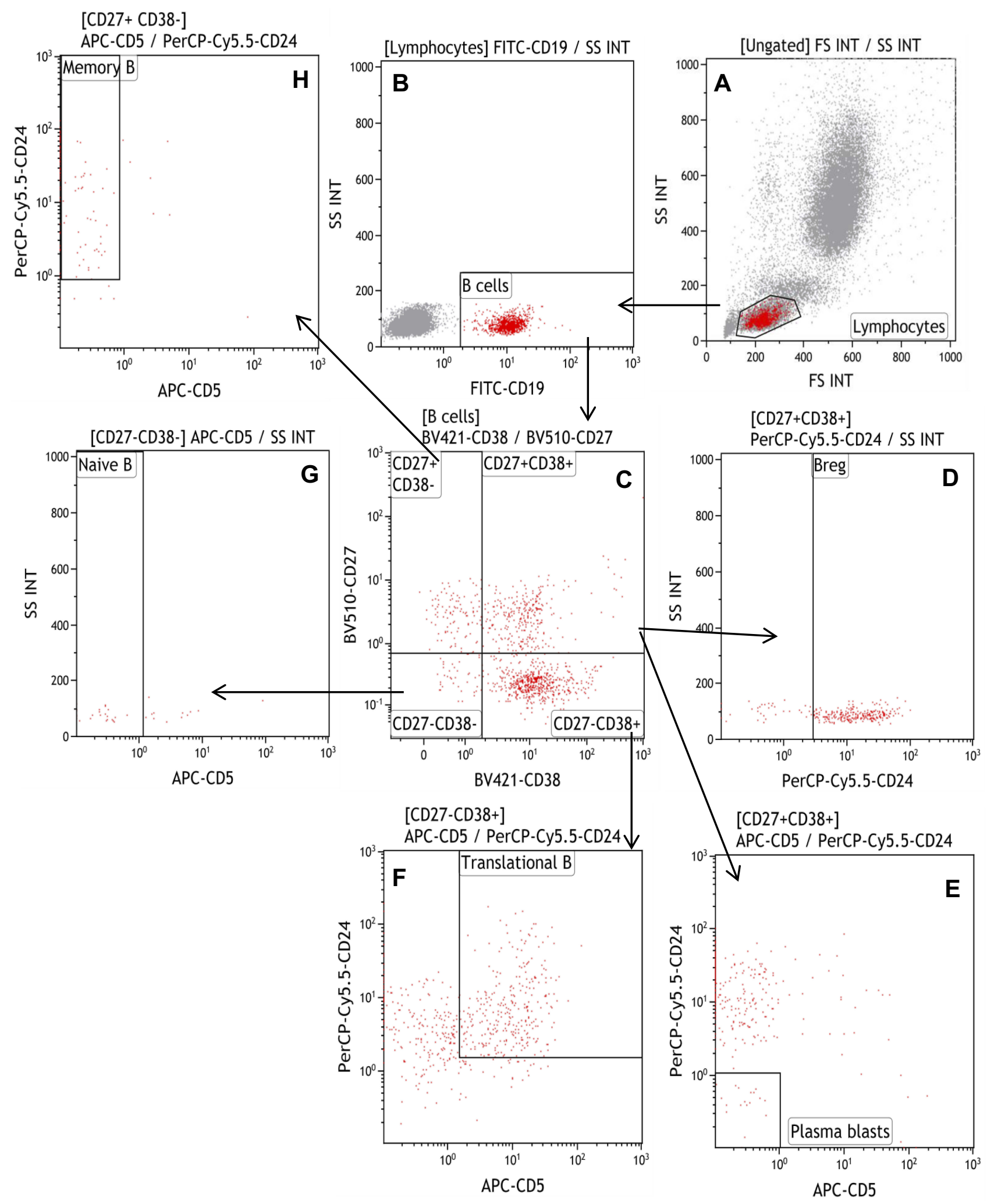

Figure 3 Gating strategy of B cell subsets (Panel 3). B cells were separated from lymphocytes (A) by CDI9 staining (B), and CD27 versus CD38 gating (C) allowed the separation of B cells, including Breg stained by CD24 (D), and without CD5 and CD24 expression on plasma blasts (E); however, CD5 and CD38 were expressed on translational B cells (F), Naïve B cells identified by negative expressions of CD27, CD38, CD5 (G), and CD24 and CD27 were expressed on memory B cells $(\mathbf{H})$. 
(CD196-CD183-), Th17 (CD196+CD183-) from Th (CD3 $+\mathrm{CD} 4+$ ), the other logical line was NK cells (CD3-CD56 + ), NKT cells (CD3+CD56+) and CD3-CD56- cells which were identified from lymphocytes. Then, NK cells were further distinguished into the CD16-NK and CD16+NK subpopulations. Meanwhile, the expression of CD16 was analyzed in CD3-CD56- cells. Panel 3 contained B cells and its 5 subsets with three logical hierarchies. B cells $(\mathrm{CD} 19+)$ separated from lymphocytes would be differentiated into Breg $(\mathrm{CD} 38+\mathrm{CD} 27+\mathrm{CD} 24+)$, Plasma blasts (CD38+CD27+CD24-CD5-), Translational B (CD38 +CD27-CD24+CD5+), Naïve B (CD38-CD27-CD5-) and
Memory B (CD38-CD27+CD24+CD5-) in terms of their differential expression of CD27, CD38, CD5 and CD24.

The percentage of $\mathrm{T} / \mathrm{B} / \mathrm{NK}$ and their subsets here referred to the percentage of each cell subset relative to lymphocytes. The relevant statistical data for each subgroup (mean \pm standard deviation (SD) and 95\% confidential interval (CI)) are shown in Table 2. The homogeneity of variance of the indicated parameters was shown by Levene test, and there was no significant statistical difference between males and females determined by independent $T$ test (Table 3). The nonparametric test showed that 29 lymphocyte subsets met

Table 2 Reference Intervals of Lymphocyte Subsets and Indicators

\begin{tabular}{|c|c|c|c|c|c|c|}
\hline \multirow[t]{2}{*}{ Parameters } & \multicolumn{2}{|l|}{ Male } & \multicolumn{2}{|l|}{ Female } & \multicolumn{2}{|l|}{ Total } \\
\hline & Mean $\pm S D$ & $95 \% \mathrm{Cl}$ & Mean \pm SD & $95 \% \mathrm{Cl}$ & Mean士SD & $95 \% \mathrm{Cl}$ \\
\hline \multicolumn{7}{|l|}{ Panel I: T cell subsets } \\
\hline DNT percentage (\%) & $3.0 \pm 0.87$ & $2.38-3.72$ & $2.9 \pm 0.86$ & $2.42-3.42$ & $3.0 \pm 0.85$ & $2.60-3.34$ \\
\hline Effector Th percentage (\%) & $0.6 \pm 0.63$ & $0.14-1.11$ & $0.7 \pm 0.58$ & $0.35-1.02$ & $0.7 \pm 0.59$ & $0.4 I-0.92$ \\
\hline EM Tc percentage (\%) & $6.4 \pm 3.13$ & $4.03-8.84$ & $5.0 \pm 3.24$ & $3.13-6.87$ & $5.6 \pm 3.21$ & $4.17-6.95$ \\
\hline Naive Tc percentage (\%) & $8.4 \pm 3.69$ & $5.57-I I .24$ & $10.0 \pm 3.89$ & $7.72-12.22$ & $9.4 \pm 3.81$ & $7.71-11.01$ \\
\hline CM Tc percentage (\%) & $3.2 \pm 1.42$ & $2.11-4.29$ & $3.5 \pm 2.44$ & $2.05-4.87$ & $3.4 \pm 2.07$ & $2.47-4.26$ \\
\hline Effector Tc percentage (\%) & $7.1 \pm 2.63$ & $5.03-9.08$ & $5.0 \pm 2.54$ & $3.54-6.48$ & $5.8 \pm 2.72$ & $4.64-6.99$ \\
\hline EM Th percentage (\%) & $4.5 \pm 3.03$ & $2.20-6.86$ & $4.3 \pm 2.7 \mid$ & $2.76-5.90$ & $4.4 \pm 2.77$ & $3.21-5.6 \mathrm{I}$ \\
\hline Naive Th percentage (\%) & $14.1 \pm 4.35$ & $10.78-17.46$ & $18.8 \pm 7.59$ & $14.40-23.16$ & $17.0 \pm 6.80$ & $14.02-19.90$ \\
\hline CM Th percentage (\%) & $18.4 \pm 6.15$ & $|3.66-23.1|$ & $|9.0 \pm 5.5|$ & $15.77-22.13$ & $18.7 \pm 5.64$ & $16.29-21.17$ \\
\hline TCR $\gamma \delta$ percentage (\%) & $3.3 \pm 0.95$ & $2.55-4.00$ & $3.2 \pm 1.03$ & $2.57-3.75$ & $3.2 \pm 0.98$ & $2.78-3.63$ \\
\hline TCR $\alpha \beta$ percentage (\%) & $62.2 \pm 5.60$ & $57.84-66.46$ & $65.8 \pm 6.35$ & $62.16-69.49$ & $64.4 \pm 6.22$ & $61.70-67.08$ \\
\hline Tc percentage $(\%)$ & $24.9 \pm 4.17$ & $21.70-28.13$ & $23.3 \pm 3.99$ & $20.99-25.59$ & $23.9 \pm 4.05$ & $22.17-25.68$ \\
\hline Th percentage $(\%)$ & $37.6 \pm 6.82$ & $32.36-42.84$ & $42.7 \pm 7.41$ & $38.4 I-46.97$ & $40.7 \pm 7.47$ & $37.47-43.93$ \\
\hline T cells percentage $(\%)$ & $66.4 \pm 5.71$ & $61.98-70.76$ & $69.7 \pm 6.16$ & $66.10-73.22$ & $68.4 \pm 6.08$ & $65.74-71.00$ \\
\hline $\mathrm{Th} / \mathrm{Tc}$ & $1.6 \pm 0.68$ & $1.07-2.12$ & $1.9 \pm 0.63$ & $1.56-2.28$ & $1.8 \pm 0.65$ & $1.5 \mathrm{I}-2.08$ \\
\hline \multicolumn{7}{|l|}{ Panel 2: NKT and Th, NK cell subsets } \\
\hline CDI6-NK percentage (\%) & $0.5 \pm 0.16$ & $0.38-0.63$ & $0.5 \pm 0.17$ & $0.42-0.61$ & $0.5 \pm 0.16$ & $0.44-0.58$ \\
\hline CDI6+NK percentage (\%) & $11.8 \pm 4.98$ & $7.93-15.59$ & $11.9 \pm 5.64$ & $8.61-15.13$ & $11.8 \pm 5.28$ & $9.54-14.11$ \\
\hline CD3-CD56-CDI6+ percentage (\%) & $3.1 \pm 1.61$ & $1.83-4.30$ & $2.6 \pm 1.58$ & $1.67-3.50$ & $2.8 \pm 1.57$ & $2.09-3.45$ \\
\hline ThI7 percentage (\%) & $9.3 \pm 5.67$ & $4.89-13.62$ & $7.8 \pm 5.26$ & $4.73-10.80$ & $8.3 \pm 5.35$ & $6.03-10.66$ \\
\hline Th2 percentage (\%) & $13.8 \pm 7.28$ & $8.20-19.39$ & $20.9 \pm 8.68$ & $15.94-25.94$ & $|8| \pm 8.74$. & $|4.37-2| .92$ \\
\hline ThI percentage (\%) & $7.7 \pm 3.65$ & $4.95-10.55$ & $13.5 \pm 8.68$ & $8.45-18.48$ & $11.2 \pm 7.58$ & $7.95-|4.5|$ \\
\hline NKT percentage (\%) & $6.1 \pm 2.07$ & $4.53-7.71$ & $4.0 \pm 2.63$ & $2.48-5.52$ & $4.8 \pm 2.60$ & $3.71-5.96$ \\
\hline NK percentage (\%) & $12.3 \pm 4.96$ & $8.45-16.08$ & $|2.4 \pm 5.7|$ & $9.09-15.69$ & $12.3 \pm 5.32$ & $10.04-14.64$ \\
\hline Treg percentage (\%) & $5.0 \pm 1.22$ & $4.10-5.98$ & $6.2 \pm 1.42$ & $5.35-6.99$ & $5.7 \pm 1.43$ & $5.11-6.35$ \\
\hline \multicolumn{7}{|l|}{ Panel 3: B cell subsets } \\
\hline Naive B percentage (\%) & $0.4 \pm 0.15$ & $0.28-0.51$ & $0.4 \pm 0.40$ & $0.19-0.65$ & $0.4 \pm 0.32$ & $0.27-0.55$ \\
\hline Breg percentage (\%) & $1.8 \pm 0.58$ & $1.35-2.24$ & $2.0 \pm 0.93$ & $1.48-2.54$ & $1.9 \pm 0.80$ & $1.58-2.27$ \\
\hline Memory B percentage (\%) & $1.0 \pm 0.77$ & $0.37-1.56$ & $0.7 \pm 0.50$ & $0.38-0.95$ & $0.8 \pm 0.62$ & $0.5 \mathrm{I}-1.05$ \\
\hline Plasma blasts percentage (\%) & $0.1 \pm 0.07$ & $0.05-0.16$ & $0.2 \pm 0.14$ & $0.10-0.26$ & $0.1 \pm 0.12$ & $0.10-0.20$ \\
\hline Translational B percentage (\%) & $2.4 \pm 1.20$ & $1.50-3.35$ & $3.7 \pm 1.71$ & $2.69-4.66$ & $3.2 \pm 1.62$ & $2.48-3.89$ \\
\hline B cells percentage $(\%)$ & $12.0 \pm 3.79$ & $9.05-14.88$ & $|3.6 \pm 3.5|$ & $11.58-15.64$ & $13.0 \pm 3.63$ & II.40-14.54 \\
\hline
\end{tabular}

Abbreviations: SD, standard deviation; $\mathrm{Cl}$, confidence interval; DNT, double-negative T cells (CD3+CD4-CD8-); EM, effector memory; CM, center memory. 
Table 3 The Correlation of Lymphocyte Subsets Between Males and Females by T-Test Analyses

\begin{tabular}{|c|c|c|c|c|}
\hline \multirow[t]{2}{*}{ Parameters } & \multicolumn{2}{|c|}{ Levene Test } & \multicolumn{2}{|l|}{$T$ test } \\
\hline & $\mathbf{F}$ & $P($ value) & $\mathbf{t}$ & P(M\&F Two Side) \\
\hline \multicolumn{5}{|l|}{ Panel I: $T$ cell subsets } \\
\hline DNT (CD3+/CD4-/CD8-) (\%) & 0.002 & 0.961 & 0.347 & 0.732 \\
\hline EffectorTh(CD3+/CD4+/CD8-/CD I97-/CD45RA+) (\%) & 0.004 & 0.948 & -0.242 & 0.811 \\
\hline EM Tc (CD3+/CD4-/CD8+/CD 197-/CD45RA-) (\%) & 0.064 & 0.803 & 1.051 & 0.305 \\
\hline Naive Tc (CD3+/CD4-/CD8+/CDI97+/CD45RA+) (\%) & 0.083 & 0.776 & -0.958 & 0.349 \\
\hline CM Tc (CD3+/CD4-/CD8+/CDI97+/CD45RA-) (\%) & 1.217 & 0.282 & -0.288 & 0.776 \\
\hline EffectorTc(CD3+/CD4-/CD8+/CD I97-/CD45RA+) (\%) & 0.272 & 0.607 & 1.856 & 0.078 \\
\hline (CD3+/CD4+/CD8-/CDI97-/CD45RA-)EM Th (\%) & 0.428 & 0.520 & 0.164 & 0.871 \\
\hline Naive Th (CD3+/CD4+/CD8-/CDI97+/CD45RA+) (\%) & 1.939 & 0.178 & -1.668 & 0.110 \\
\hline CM Th(CD3+/CD4+/CD8-/CD 197+/CD45RA-) (\%) & 0.303 & 0.588 & -0.230 & 0.821 \\
\hline $\mathrm{TCR} \gamma \delta(\mathrm{CD} 3+/ \mathrm{TCR} \alpha \beta-/ \mathrm{TCR} \gamma \delta+)(\%)$ & 0.014 & 0.908 & 0.261 & 0.796 \\
\hline TCR $\alpha \beta(\mathrm{CD} 3+/ \mathrm{TCR} \alpha \beta+/ \mathrm{TCR} \gamma \delta-)(\%)$ & 0.006 & 0.938 & $-1.4 \mid 4$ & 0.172 \\
\hline Tc(CD3+/CD4-/CD8+) (\%) & 0.025 & 0.876 & 0.938 & 0.359 \\
\hline $\operatorname{Th}(C D 3+/ C D 4+/ C D 8-)$ (\%) & 0.092 & 0.764 & -1.657 & 0.112 \\
\hline T cells(CD3+) $(\%)$ & 0.203 & 0.657 & -1.285 & 0.213 \\
\hline $\mathrm{Th} / \mathrm{Tc}$ & 0.079 & $0.78 \mathrm{I}$ & -1.166 & 0.257 \\
\hline \multicolumn{5}{|l|}{ Panel 2: NKT and Th, NK cell subsets } \\
\hline CDI6-NK(CD3-/CD56+/CDI6-) (\%) & 0.152 & 0.701 & -0.128 & 0.900 \\
\hline CDI6+NK(CD3-/CD56+/CDI6+) (\%) & 0.044 & 0.836 & -0.049 & 0.961 \\
\hline CD3-CD56-CDI6+ (\%) & 0.278 & 0.603 & 0.710 & 0.486 \\
\hline ThI7(CD3+/CD4+/CDI83-/CDI96+) (\%) & 0.198 & 0.661 & 0.645 & 0.526 \\
\hline Th2(CD3+/CD4+/CDI83-/CDI96-) (\%) & 0.769 & 0.390 & -2.052 & 0.053 \\
\hline ThI (CD3+/CD4+/CDI83+/CDI96-) (\%) & 3.162 & 0.090 & -1.861 & 0.077 \\
\hline NKT(CD3+/CD56+) (\%) & 0.318 & 0.579 & 2.035 & 0.055 \\
\hline NK (CD3-/CD56+) (\%) & 0.029 & 0.866 & -0.052 & 0.959 \\
\hline Treg (CD3+/CD4+/CD25+/CD 127dim/-) (\%) & 0.450 & 0.510 & -1.960 & 0.063 \\
\hline \multicolumn{5}{|l|}{ Panel 3: B cell subsets } \\
\hline Naive B (CDI9+/CD27-/CD38-/CD5-) (\%) & 1.118 & 0.302 & -0.208 & 0.838 \\
\hline Breg (CD19+/CD27+/CD38+/CD24+) (\%) & 1.910 & 0.181 & -0.621 & 0.541 \\
\hline Memory B (CD19+/CD27+/CD38-/CD5-/CD24+) (\%) & 2.511 & 0.128 & 1.136 & 0.269 \\
\hline Plasma blasts (CD19+/CD27+/CD38+/CD5-/CD24-) (\%) & 1.394 & 0.251 & -1.433 & 0.166 \\
\hline Translational B(CD19+/CD27-/CD38+/CD5+/CD24+) (\%) & 0.712 & 0.408 & -1.906 & 0.070 \\
\hline B cells percentage $(C D \mid 9+)(\%)$ & 0.248 & 0.624 & -1.064 & 0.299 \\
\hline
\end{tabular}

Abbreviations: SD, standard deviation; $\mathrm{Cl}$, confidence interval; DNT, double-negative T cells (CD3+CD4-CD8-); EM, effector memory; CM, center memory.

the requirements of a normal distribution, no matter whether they were from male or female or whether they were part of cancer patients or healthy donors. The data were shown as the mean, SD and $95 \%$ confidential interval (CI) for male, female and both genders were presented by one-sample $t$-test.

\section{Expression of Lymphocyte Subsets in Patients with Malignant Solid Tumors}

The Mann-Whitney $U$-test model was used to analyze the differences of the percentages and ratios of 29 lymphocyte subsets between healthy donors and cancer patients that did not coincide with normal distribution analyzed by the non-parametric test. As shown in Figure 4 and Table 4, the proportions of Breg $(p<0.001)$ and CD16-NK cells $(p<0.001)$ were significantly lower than that of a healthy control group. And a similar phenomenon was also occurred in the group of Naïve Th $(p<0.01)$, Naïve Tc $(p<0.01)$, CM Tc $(p<0.01)$ and B cells $(p<0.05)$. However, patients with malignant solid tumors expressed significantly higher proportions of EM Th $(p<0.001)$, EM Tc $(p<0.01)$, Effector Tc $(p<0.01)$, Treg $(p<0.05)$ and Tc $(p<0.05)$. 

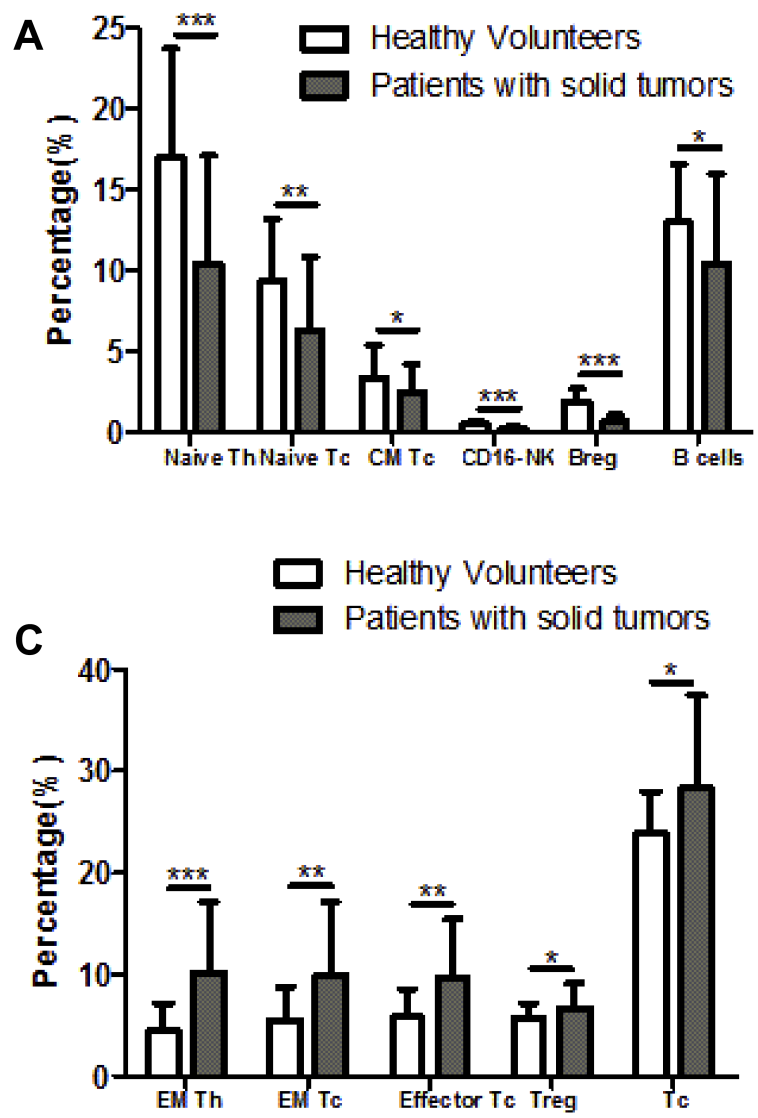
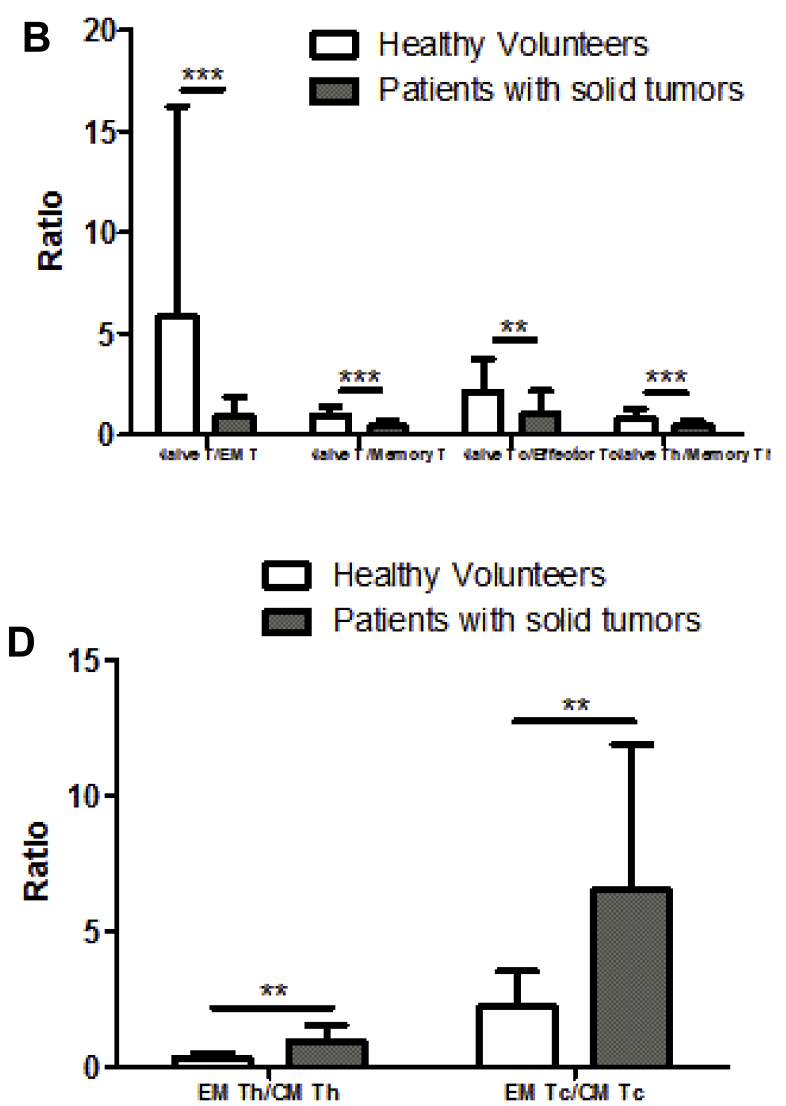

Figure 4 Differences in Lymphocyte subsets between patients with malignant solid tumors and healthy donors. There were decreased percentages or ratios of Naive Th, Naïve Tc, CM Tc, CDI6-NK, Breg, B cells (A) and Naïve T/EM T, Naïve T/Memory T, Naïve Tc/Effector Tc, Naïve Th/Naïve Tc (B) in patients with solid tumors compared with healthy donors. However, there were increased percentages or ratios of EM Th, EM Tc, Effector Tc, Treg, Tc (C) and EM Th/CM Th, EM Tc/CM Tc (D) in patients with solid tumors compared to healthy donors. ${ }^{*} \mathrm{P}<0.05$, $* * \mathrm{P}<0.01$, $* * * \mathrm{P}<0.001$.

\section{The Ratio of Peripheral T Cell}

\section{Characteristics in Patients with Malignant} Solid Tumors

This study demonstrated that the Naive Th/Memory Th ratio $(p<0.001)$ was significantly lower in patients with malignant solid tumors. Similarly, the ratios of both Naïve T cells/Memory T cells $(p<0.001)$ and Naïve $\mathrm{T}$ cells/EM T cells $(p<0.001)$ in cancer patients were relatively lower. However, cancer patients exhibited considerably higher EM Th cells/CM Th cell ratio $(p<0.01)$ and EM Tc cells/CM Tc cell ratio $(p<0.01)$ than healthy donors. The Th1/Th2 ratio $(p>0.05)$ and Th17/Treg $(p>0.05)$ had no statistical significance (data not shown).

\section{Discussion}

During cancer progression, the tumor microenvironment is crucial in modulating immune responses. ${ }^{15}$ Innate and adaptive immunity played important roles in cancer development and were closely correlated with cancer therapeutics. ${ }^{16}$ Measurement of immune function and status for cancer patients is an important supplementary diagnostic method in clinics. As far as we know, this is a study with the largest amounts of parameters for lymphocyte subsets to measure the immune system of patients with malignant solid tumors and to investigate the clinical significance of T, B, NK lymphocyte subsets and their ratios.

Memory $\mathrm{T}$ cells attack tumor cells, which elicits a robust immune response in tumor tissues. ${ }^{17}$ In contrast, naïve $\mathrm{T}$ cells played an important role in proliferation and anti-tumor efficiency. ${ }^{6}$ The clinical significance of peripheral Naive Th cells/Memory Th cells, Naïve Th cells and Naïve Tc cells was consistent with the previous findings in NSCLC, ${ }^{12,18}$ which illustrated that not only were these indicators useful for the prediction and prognosis of NSCLC, but also had the clinical significance in patients with malignant solid tumors. Furthermore, we found the decreased peripheral ratios of Naïve $\mathrm{T}$ cells/Memory T cells, Naïve T cells/EM T cells and percentage of CM Tc cells in cancer patients. Meanwhile, the increased 
Table 4 Differences in Lymphocyte Subsets Between Patients with Malignant Solid Tumors and Healthy Donors

\begin{tabular}{|l|l|l|l|}
\hline Variable & $\begin{array}{l}\text { Healthy } \\
\text { Volunteer }\end{array}$ & $\begin{array}{l}\text { Patients } \\
\text { with Solid } \\
\text { Tumors }\end{array}$ & \\
\cline { 2 - 3 } & Mean \pm SD & Mean \pm SD & \\
\hline Breg percentage (\%) & $1.9 \pm 0.80$ & $0.6 \pm 0.42$ & $<0.001$ \\
CD3-CD56+CDI6- & $0.5 \pm 0.16$ & $0.2 \pm 0.12$ & $<0.001$ \\
percentage (\%) & & & \\
EM Th percentage (\%) & $4.4 \pm 2.77$ & $10.2 \pm 6.93$ & $<0.001$ \\
Naïv Th/Memory Th & $0.8 \pm 0.47$ & $0.4 \pm 0.31$ & $<0.001$ \\
Naïv T/EM T & $5.8 \pm 10.45$ & $0.9 \pm 1.01$ & $<0.001$ \\
Naïv T/Memory T & $0.9 \pm 0.50$ & $0.4 \pm 0.33$ & $<0.001$ \\
Naïve Tc/Effector Tc & $2.1 \pm 1.66$ & $1.0 \pm 1.18$ & 0.001 \\
Naive Th percentage (\%) & $17.0 \pm 6.80$ & $10.4 \pm 6.76$ & 0.001 \\
EM Th/CM Th & $0.3 \pm 0.19$ & $0.9 \pm 0.60$ & 0.002 \\
EM Tc/CM Tc & $2.2 \pm 1.32$ & $6.5 \pm 5.40$ & 0.003 \\
Naive Tc percentage (\%) & $9.4 \pm 3.81$ & $6.3 \pm 4.52$ & 0.004 \\
Effector Tc percentage (\%) & $5.8 \pm 2.72$ & $9.6 \pm 5.82$ & 0.005 \\
EM Tc percentage (\%) & $3.4 \pm 2.07$ & $10.0 \pm 7.20$ & 0.006 \\
CM Tc percentage (\%) & $3.4 \pm 2.07$ & $2.5 \pm 1.72$ & 0.019 \\
B cells percentage (\%) & $13.0 \pm 3.63$ & $10.4 \pm 5.60$ & 0.014 \\
Treg percentage (\%) & $5.7 \pm 1.43$ & $6.7 \pm 2.38$ & 0.026 \\
Tc percentage (\%) & $23.9 \pm 4.05$ & $28.3 \pm 9.13$ & 0.046 \\
\hline
\end{tabular}

Abbreviations: SD, standard deviation; EM, effector memory; CM, center memory.

peripheral ratios of EM Th cells/CM Th, EM Tc cells/CM Tc cells and percentages of EM Th cells, EM Tc and effector Tc cells were determined in a patient with malignant solid tumors. As the primary immune cells, $\mathrm{T}$ lymphocytes had a remarkable diagnostic and therapeutic utility in anti-tumor response. In normal T cell physiology, there are naturally occurring "off signals" to ensure appropriate control of the robust and cascading $\mathrm{T}$ cell responses. ${ }^{19}$ Herein, the indicators we showed in this study could reveal the variation trends of $\mathrm{T}$ lymphocyte subsets dynamically and precisely. In healthy individuals, Treg cells played an important role in the maintenance of self-tolerance. From the published data, increased Treg cells in various tumors have been observed. ${ }^{20,21}$ The balance of Treg/Th17 was closely related to immunity and immune tolerance. The Th17/Treg imbalance played a central role in disease pathogenesis. ${ }^{22} \mathrm{~A}$ recent study displayed the increased proportions of Treg cells in NSCLC patients, and this may indicate tumor progression. ${ }^{23}$ Another study observed that Treg cells, Th17 cells and Treg/Th17 ratio were increased in HCC patients. Treg/Th17 imbalance might serve as an important indicator for determining the progression and prognosis of
HCC. ${ }^{13}$ The present study found that the proportion of Treg cells was significantly higher in patients with solid tumors than that in the healthy control group. But the proportions of Th17 cells and the ratio of Treg/Th17 had no statistical significance between patients with malignant solid tumors and healthy donors, which may be not consistent with the previous study of HCC. ${ }^{13}$ We speculated that different methods for pretreatment and analysis may influence the results. In addition, our study consisted of limited cohorts and the cancer patients in the study were not homogeneous.

B cells played an important role in modulating antitumor immunity. ${ }^{24,25}$ The changes of Breg and B cells found in patients with malignant solid tumors are also consistent with previous findings in patients with melanoma, lung adenocarcinoma. ${ }^{26}$ But another study observed increased $\mathrm{CD} 19+/ \mathrm{CD} 24^{\mathrm{hi}} / \mathrm{CD} 38^{\mathrm{hi}}$ Breg cells in gastric cancer. ${ }^{27}$ We assumed that different markers may affect the results. In previous studies, the most appropriate markers for Breg cells were not in consensus, and therefore, different research teams have used distinct surface and intracellular markers of regulatory B cells. ${ }^{28-30}$

As an immature NK cell subset, peripheral CD16-NK cells can lead to cytokine production ${ }^{31}$ and decreased remarkedly in patients with malignant solid tumors, which suggests the tumor burden suppressed the function of CD16-NK cells.

The limitations of our study are the small numbers of healthy donors for calculating reference intervals, as well as heterogeneous patient cohorts with different clinical stages and treatment strategies. Therefore, we intend to take advantage of the controls with inflammation or autoimmune diseases to further verify our preliminary findings in future studies, and to verify the predictive and prognostic role of these indicators before and after treatment with cell therapy.

The Science journal has unveiled "cancer immunotherapy" as one of the ten breakthroughs in $2013 .{ }^{32}$ The newly developed therapies, such as "checkpoint blockade" and "CAR-T" (chimeric-antigen receptor $\mathrm{T}$ cell), which are aimed to prevent $\mathrm{T}$ cell immunosuppression, have demonstrated impressive clinical outcomes in both solid ${ }^{33,34}$ and hematologic malignancies. ${ }^{34}$ CART19 (Kymriah, Novartis) and KTE-C19 (Yescartais, Kit Pharma) are newly the FDAapproved drugs, but both of them are used for the treatment of hematologic malignancies.

In summary, the above indicators, especially the ratio and proportion of Naive T cells, Memory T cells and their subsets, 
may play an important role in the prediction and prognosis of CAR-T therapy for patients with malignant solid tumors.

\section{Ethics Approval and Consent to Participate}

This study was conducted in accordance with the regulations on patient confidentiality and the ethical standards of Declaration of Helsinki. The present study was approved by the ethics committees of Eastern Hepatobiliary Surgery Hospital in Shanghai, China and performed in accordance with relevant guidelines and regulations.

\section{Data Sharing Statement}

The datasets used and/or analysed during the current study are available from the corresponding authors on reasonable request.

\section{Author Contributions}

Zhenlong Ye, Fuping Zhou, Sufang Zhang, Qijun Qian designed the project, Zhi Zhu, Na Ding, Fuping Zhou, Jinxing Lou and Jinrong Qiu recorded the information of patients and collected the samples, Jinrong Qiu, Fuping Zhou, Xinchun Li, Sufang Zhang, Zhuo Chen, Gaoxiong Lu conducted the experiments, Xinchun Li, Sufang Zhang, Zhuo Chen, and Shuo Ma wrote the manuscript, Zhenlong Ye, Zhuo Chen, Shuo Ma, Zenghui Xu revised the manuscript, Jinxing Lou, Jinrong Qiu and Qijun Qian supported the project. All authors contributed to data analysis, drafting or revising the article, gave final approval of the version to be published, and agreed to be accountable for all aspects of the work.

\section{Funding}

This work was supported by the National Key Research and Development Program (2017YFC0909800), the National Science Fund Projects (8167110226, 81703047), Shanghai Science and Technology Development Funds (19QB1405900), Scientific Research Project of Jiading Health and Family Planning Commission (2018-QN-13), the study on the application system of tumor intelligence precision medical treatment based on large health data of Shanghai Informatization Development Special Project (201602037) and the Capacity Building Project of Shanghai Engineering Research Center (16DZ2281000).

\section{Disclosure}

The authors report no conflicts of interest in this work.

\section{References}

1. Gomes AL, Teijeiro A, Burén S, et al. Metabolic inflammation-associated IL-17A causes non-alcoholic steatohepatitis and hepatocellular carcinoma. Cancer Cell. 2016;30(1):161-175.

2. Huang CY, Wang Y, Luo GY, et al. Relationship between PD-L1 expression and CD8+ T-cell immune responses in hepatocellular carcinoma. J Immunother. 2017;40(9):323-333. doi:10.1097/CJI.000 0000000000187

3. Barsoum IB, Smallwood CA, Siemens DR, et al. A mechanism of hypoxia-mediated escape from adaptive immunity in cancer cells. Cancer Res. 2014;74(3):665-674. doi:10.1158/0008-5472.CAN-13-0992

4. Robertson FC, Berzofsky JA, Terabe M. NKT cell networks in the regulation of tumor immunity. Front Immunol. 2014;5:543. doi:10.3389/fimmu.2014.00543

5. Dugnani E, Pasquale V, Bordignon $C$, et al. Integrating $T$ cell metabolism in cancer immunotherapy. Cancer Lett. 2017;411:12-18. doi:10.1016/j.canlet.2017.09.039

6. Hinrichs CS, Borman ZA, Gattinoni L, et al. Human effector CD8+ $\mathrm{T}$ cells derived from naive rather than memory subsets possess superior traits for adoptive immunotherapy. Blood. 2011;117(3):808-814. doi:10.1182/blood-2010-05-286286

7. Singh A, Schabath R, Ratei R, et al. Peripheral blood sCD3(-) CD4 $(+) \mathrm{T}$ cells: a useful diagnostic tool in angioimmunoblastic $\mathrm{T}$ cell lymphoma. Hematol Oncol. 2014;32(1):16-21. doi:10.1002/hon. v32.1

8. Awad MM, Jones RE, Liu H, et al. Cytotoxic T cells in PD-L1positive malignant pleural mesotheliomas are counterbalanced by distinct immunosuppressive factors. Cancer Immunol Res. 2016;4 (12):1038-1048. doi:10.1158/2326-6066.CIR-16-0171

9. Zhu P, Hu C, Hui K, Jiang X. The role and significance of VEGFR2 $(+)$ regulatory $\mathrm{T}$ cells in tumor immunity. Onco Targets Ther. 2017;10:4315-4319. doi:10.2147/OTT.S142085

10. Zhang Y, Gallastegui N, Rosenblatt JD. Regulatory B cells in anti-tumor immunity. Int Immunol. 2015;27(10):521-530. doi:10.1093/intimm/ dxv034

11. Saavedra D, García B, Lorenzo-Luaces P, et al. Biomarkers related to immunosenescence: relationships with therapy and survival in lung cancer patients. Cancer Immunol Immunother. 2016;65(1):37-45. doi:10.1007/s00262-015-1773-6

12. Peng Y, Ma J, Yang X, et al. Peripheral CD4+ naive/memory ratio is an independent predictor of survival in non-small cell lung cancer. Oncotarget. 2017;8(48):83650-83659. doi:10.18632/oncotarget. 19330

13. Lan YT, Fan X-P, Fan Y-C, et al. Change in the Treg/Th17 cell imbalance in hepatocellular carcinoma patients and its clinical value. Medicine (Baltimore). 2017;96(32):e7704. doi:10.1097/MD.0000000000007704

14. Chang Q, Hedley D. Emerging applications of flow cytometry in solid tumor biology. Methods. 2012;57(3):359-367. doi:10.1016/j. ymeth.2012.03.027

15. Grivennikov SI, Greten FR, Karin M. Immunity, inflammation, and cancer. Cell. 2010;140(6):883-899. doi:10.1016/j.cell.2010.01.025

16. Vesely MD, Kershaw MH, Schreiber RD, et al. Natural innate and adaptive immunity to cancer. Annu Rev Immunol. 2011;29:235-271. doi:10.1146/annurev-immunol-031210-101324

17. Farber DL, Yudanin NA, Restifo NP. Human memory T cells: generation, compartmentalization and homeostasis. Nat Rev Immunol. 2014;14(1):24-35. doi:10.1038/nri3567

18. Hishiki T, Mise N, Harada K, et al. Frequency and proliferative response of circulating invariant natural killer $\mathrm{T}$ cells in pediatric patients with malignant solid tumors. Pediatr Surg Int. 2018;34 (2):169-176. doi:10.1007/s00383-017-4185-1

19. Singh N, Shi J, June CH, Ruella M. Genome-editing technologies in adoptive T cell immunotherapy for cancer. Curr Hematol Malig Rep. 2017;12(6):522-529. doi:10.1007/s11899-017-0417-7 
20. Erfani N, Mehrabadi SM, Ghayumi MA, et al. Increase of regulatory $\mathrm{T}$ cells in metastatic stage and CTLA-4 over expression in lymphocytes of patients with non-small cell lung cancer (NSCLC). Lung Cancer. 2012;77(2):306-311. doi:10.1016/j.lungcan.2012.04.011

21. Ichihara F, Kono K, Takahashi A, et al. Increased populations of regulatory $\mathrm{T}$ cells in peripheral blood and tumor-infiltrating lymphocytes in patients with gastric and esophageal cancers. Clin Cancer Res. 2003;9(12):4404-4408.

22. Diller ML, Kudchadkar RR, Delman KA, et al. Balancing inflammation: the link between Th17 and regulatory T cells. Mediators Inflamm. 2016;2016:6309219. doi:10.1155/2016/6309219

23. Liu C, Wu S, Meng X, et al. Predictive value of peripheral regulatory $\mathrm{T}$ cells in non-small cell lung cancer patients undergoing radiotherapy. Oncotarget. 2017;8(26):43427-43438. doi:10.18632/oncotarget.15238

24. Yang C, Lee H, Pal S, et al. B cells promote tumor progression via STAT3 regulated-angiogenesis. PLoS One. 2013;8(5):e64159. doi:10.1371/journal.pone.0064159

25. Sarvaria A, Madrigal JA, Saudemont A. B cell regulation in cancer and anti-tumor immunity. Cell Mol Immunol. 2017;14(8):662-674. doi:10.1038/cmi.2017.35

26. DeFalco J, Harbell M, Manning-Bog A, et al. Non-progressing cancer patients have persistent $\mathrm{B}$ cell responses expressing shared antibody paratopes that target public tumor antigens. Clin Immunol. 2018;187:37-45. doi:10.1016/j.clim.2017.10.002

27. Wang WW, Yuan XL, Chen H, et al. CD19+CD24hiCD38hiBregs involved in downregulate helper $\mathrm{T}$ cells and upregulate regulatory $\mathrm{T}$ cells in gastric cancer. Oncotarget. 2015;6(32):33486-33499. doi:10.18632/oncotarget.5588

28. Noh J, Noh G, Kim HS, et al. Allergen-specific responses of CD19 $(+) \mathrm{CD} 5(+) \mathrm{Foxp} 3(+)$ regulatory B cells (bregs) and CD4(+)Foxp3(+) regulatory $\mathrm{T}$ cell (tregs) in immune tolerance of cow milk allergy of late eczematous reactions. Cell Immunol. 2012;274(1-2):109-114. doi:10.1016/j.cellimm.2012.01.005
29. Blair PA, Noreña LY, Flores-Borja F, et al. CD19(+)CD24(hi) CD38(hi) B cells exhibit regulatory capacity in healthy individuals but are functionally impaired in systemic lupus erythematosus patients. Immunity. 2010;32(1):129-140. doi:10.1016/j.immuni.20 09.11 .009

30. Aybar LT, McGregor JG, Hogan SL, et al. Reduced CD5(+) CD24(hi) CD38(hi) and interleukin-10(+) regulatory $\mathrm{B}$ cells in active anti-neutrophil cytoplasmic autoantibody-associated vasculitis permit increased circulating autoantibodies. Clin Exp Immunol. 2015;180 (2):178-188. doi:10.1111/cei.12483

31. Amand M, Iserentant G, Poli A, et al. Human CD56(dim)CD16(dim) cells as an individualized natural killer cell subset. Front Immunol. 2017;8:699. doi:10.3389/fimmu.2017.00699

32. Couzin-Frankel J; Breakthrough of the year 2013. Cancer immunotherapy. Science. 2013;342(6165):1432-1433. doi:10.1126/ science.342.6165.1432

33. Carbone DP, Reck M, Paz-Ares L, et al. First-line nivolumab in stage IV or recurrent non-small-cell lung cancer. $N$ Engl J Med. 2017;376 (25):2415-2426. doi:10.1056/NEJMoa1613493

34. Eggermont AM, Chiarion-Sileni V, Grob -J-J, et al. Prolonged survival in stage III melanoma with ipilimumab adjuvant therapy. $N$ Engl J Med. 2016;375(19):1845-1855. doi:10.1056/NEJMoa16 11299

35. Qin L, Jing X, Qiu Z, et al. Aging of immune system: immune signature from peripheral blood lymphocyte subsets in 1068 healthy adults. Aging (Albany NY). 2016;8(5):848-859. doi:10.18632/aging. v8i5

36. Henny J, Vassault A, Boursier G, et al. Recommendation for the review of biological reference intervals in medical laboratories. Clin Chem Lab Med. 2016;54(12):1893-1900. doi:10.1515/cclm-20160793
Cancer Management and Research

\section{Publish your work in this journal}

Cancer Management and Research is an international, peer-reviewed open access journal focusing on cancer research and the optimal use of preventative and integrated treatment interventions to achieve improved outcomes, enhanced survival and quality of life for the cancer patient.

\section{Dovepress}

The manuscript management system is completely online and includes a very quick and fair peer-review system, which is all easy to use. Visit http://www.dovepress.com/testimonials.php to read real quotes from published authors. 